\title{
El Estudio Flexner y una evaluación independiente de los postgrados de medicina
}

\author{
The Flexner Study and an independent evaluation of medical graduate degrees
}

\author{
José Ranulfo Lizardo Barahona, ${ }^{1}$ José Edmundo Lizardo Wildt. ${ }^{2}$
}

${ }^{1}$ Médico Especialista en Cirugía Pediátrica; Honduras Medical Center; ${ }^{2}$ Estudiante de Octavo Año, Carrera de Medicina, Universidad Católica de Honduras "Nuestra Señora Reyna de la Paz"; Tegucigalpa.

Abraham Flexner nació el 13 de noviembre de 1866 en Louisville, Estados Unidos. Completó la Licenciatura en Clásicos en la Universidad Johns Hopkins en 1886, continuó estudios con un máster en educación en Harvard para luego continuar un año de Filosofía y Sicología en la Universidad de Heidelberg, Alemania. Henry Prichett, Presidente de la Fundación Carnegie Illuminati para el Avance de la Enseñanza, en 1908 contrató a Flexner para que efectuara un estudio sobre la educación médica y las escuelas de medicina. Por esta razón Flexner visitó 155 escuelas de Medicina en 40 estados de Norteamérica, que concluyó con el informe que se publicó en 1910 con el titulo "Medical Education in United States and Canada". Conocido también como el Boletín Número Cuatro de la Fundación Carnegie Illuminati para el progreso de la enseñanza que aun hoy es la publicación mas citada sobre la educación médica. ${ }^{1-3}$

Los datos que se obtuvieron durante las visitas de campo, incluyeron la población escolar, los requisitos de ingreso, la planta docente, las instalaciones hospitalarias, instalaciones de laboratorio, los servicios educativos disponibles, los recursos financieros con que se contaba; además, la relación de la escuela con la universidad y el sistema educativo del Estado. Para cada estado o ciudad se consideraron los datos demográficos y un análisis de las condiciones en las que se daba la educación médica. El informe registró la existencia de 24,000 estudiantes, con 3,500 profesores. Además, descubrió con precisión la terrible heterogeneidad que incluía desde escuelas prestigiadas bien organizadas con todas las instalaciones y recursos necesarios como Johns Hopkins y Pennsylvania hasta el caso de escuelas que no reunían ni los requisitos mínimos para merecer tal denominación como el caso de Mississippi y Oregon. Únicamente el $20 \%$ de las Universidades pudo cumplir con las prescripciones y restricciones de Flexner. ${ }^{1-3}$

Como consecuencia del informe, un número significativo de escuelas de medicina fueron cerradas mientras otras debie-

Recibido: 25-05-2020 Aceptado para publicación 15-12-2020

Dirección para correspondencia: Dr. José Ranulfo Lizardo Barahona

Correo electrónico: ijlizardob@hotmail.com

Declaración de relaciones y actividades financieras y no financieras y conflictos de interés: ninguno.

DOI: https://doi.org/10.5377/rmh.v88i2.11500 ron realizar importantes cambios. En Estados Unidos, el número de escuelas disminuyó de 131 a 81 . El informe consta de dos partes. En la primera parte presenta una descripción de cada una de las escuelas con las razones para su mejoramiento 0 bien el cierre para las que no reunían las condiciones mínimas pertinentes para impartir la enseñanza. En la segunda parte se establecen las recomendaciones de las cuales mencionaremos las importantes: adopción de requisitos de ingreso más exigentes sobre todo en ciencias y matemáticas, un currículo de cuatro años para estudiar medicina, dos años de ciencias básicas y dos años de enseñanza clínica en hospitales, vinculación permanente de la escuela de medicina con la universidad con una mayor productividad científica y de investigación. Además, la creación de un Consejo Nacional de Examinadores Médicos que otorgaran únicamente a quienes alcanzaran los requisitos académicos un Diploma de Certificación que serían estatales. Es decir, un ente independiente de la universidad que otorgaría la autorización para ejercer la medicina en cada uno de los estados. Obviamente la implementación de todos estos cambios requería que los profesores de medicina fueran educadores a tiempo completo, para lo cual Flexner proponía un aumento de sueldo de 5,000 dólares anuales a 20,000 dólares, lo que les permitiría la dedicación exclusiva al hospital universitario como docentes y como investigadores ${ }^{1-5}$ De tal manera que el Informe Flexner transformó radicalmente la educación médica de pregrado y postgrado, reglamentando como debía educarse a los médicos; tanto así que la medicina occidental como es hoy en día en gran medida se debe a dicho estudio.

Para la mayoría de los países, las áreas del conocimiento para generar bienestar a la población involucran una inversión grande en sumas de dinero para capacitar el talento humano con el fin de cubrir las necesidades de salud del país. En este sentido los gobiernos tienen dos alternativas. La primera es invertir para formar el talento humano en su propio país y la segunda es invertir para una formación en el exterior. Es importante recalcar que la formación de profesionales en el área de la medicina está inmersa en el proceso de enseñanza, aprendizaje y evaluación en el que es necesario garantizar el desarrollo de habilidades confiables con las competencias específicas, por lo cual es preciso un proceso de carácter tutelar. La competen- 
cia debe ser entendida como una adquisición de habilidades que le permitan al médico realizar un conjunto de tareas con un estándar apropiado.

En nuestro país, hace 45 años se iniciaron los posgrados en medicina; incluso en la última década se incorporaron las subespecialidades sobretodo en pediatría y cirugía. ${ }^{6}$ Sin embargo, durante todo este tiempo, el único conocimiento que tiene la sociedad con respecto a estos programas es el que deriva de la información que la misma institución proporciona y aun cuando sabemos que estas instituciones educativas son particularmente sensibles a las críticas externas, es evidente que una investigación imparcial requiere que la misma sea externa. Si somos honestos tendremos que reconocer que nuestra realidad es compatible con la descripción flexneriana ya que aún hoy, 110 años después, no tenemos profesores a tiempo completo, no hay una producción científica ni de investigación y no existe un consejo nacional de examinadores médicos independientes que otorgue un diploma de certificación. ${ }^{7}$ La simple verificación del financiamiento del carácter estructural de las instalaciones, los requisitos del ingreso, la plantilla de profesores a tiempo completo, la gestión del programa, el número de autopsias, de sesiones clínico patológicas, la tasa de investigación y de publicaciones, nos proporcionará la información pertinente siempre y cuando sea un proceso independiente e imparcial. No queremos adelantarnos a los resultados, pero es muy posible que las recomendaciones sean similares al estudio Flexner de 1910. Entendemos que la solicitud no será de la aceptación de la mayoría; sin embargo, por el bien de la educación médica y de la sociedad en Honduras, es lo que procede.

\section{CONTRIBUCIÓN}

Ambos autores participaron en la concepción de la idea original del artículo, en la búsqueda bibliográfica y redacción del mismo. Ambos autores procedieron con las recomendaciones editoriales y aprobaron la versión final del artículo.

\section{REFERENCIAS}

1. Bliss M. William Osler: Una vida entregada a la medicina. $2^{\mathrm{a}}$ ed. Madrid: Ergon; 2006

2. Narro J. III. La Herencia de Flexner. Las Ciencias Básicas, el Hospital, el Laboratorio, la Comunidad Gac. Med. Mex. 2004;140(1):52-55. .

3. Vicedo A. Abraham Flexner, pionero de la educación médica. Educ. Med. Super.[Internet]. 2002[citado 05 mayo 2020];16(2):156-163. Disponible en: $\quad$ http://scielo.sld.cu/scielo.php?script=sci_arttext\&pid=s086421412002000200010\&lng=es\&nrm=iso>.ISSN0864-2141.

4. Martini CJ. The Long Shadow of Flexner: a prolonged polemic in assessing outcomes in medical education. JAMA. 1989;262(8):1008-1010

5. Fisher JE. Flexner and the whole- time system: the second Flexner report and the whole-time system in American academic surgery. AM J Surg. 1999;178(1): 2-3.

6. Lizardo JR. Los Maestros que necesitamos. Rev Méd Hondur. 2016; 84: 72.

7. Solano Velásquez JS. Percepción de factores modificables que afectan la educación médica en Honduras: Encuesta 2019. Rev Méd Hondur. 2019; 87: 55-62. 